\title{
Miranda
}

Revue pluridisciplinaire du monde anglophone /

Multidisciplinary peer-reviewed journal on the English-

speaking world

15 | 2017

Lolita at 60 / Staging American Bodies

\section{Les « Variations Dolores » - 2010-2016}

Nouvelles lectures-réécritures de Lolita

\section{Yannicke Chupin}

\section{OpenEdition}

\section{Journals}

Édition électronique

URL : http://journals.openedition.org/miranda/11163

DOI : 10.4000/miranda. 11163

ISSN : 2108-6559

\section{Éditeur}

Université Toulouse - Jean Jaurès

Référence électronique

Yannicke Chupin, «Les « Variations Dolores »- 2010-2016», Miranda [En ligne], 15 | 2017, mis en ligne le 06 octobre 2017, consulté le 16 février 2021. URL : http://journals.openedition.org/miranda/11163 ; DOI : https://doi.org/10.4000/miranda.11163

Ce document a été généré automatiquement le 16 février 2021.

\section{(c) $(1) \&$}

Miranda is licensed under a Creative Commons Attribution-NonCommercial-NoDerivatives 4.0 International License. 


\title{
Les « Variations Dolores »- 2010-2016
}

\author{
Nouvelles lectures-réécritures de Lolita
}

Yannicke Chupin

1 Au colloque de la Société française Vladimir Nabokov à Biarritz, en avril 2016, le pionnier des études nabokoviennes françaises, Maurice Couturier, dévoilait le titre de son prochain roman: Le Rapt de Lolita. Quelques mois plus tard, en novembre 2016, paraissait un recueil de nouvelles, intitulé Lolita, Variations sur un Thème, fruit d'un collectif d'écrivains français réinterprétant le mythe de Lolita aujourd'hui ${ }^{1}$. La métaphore musicale, qui constituait également le sous-titre donné par l'écrivain suédoise Sara Stridsberg à sa lecture-réécriture de Lolita-Darling River, Les Variations Dolores-traduit la double polarité de toute réécriture. «On ne peut varier sans répéter, ni répéter sans varier », souligne Genette $(1999,101)^{2}$. La variation implique la reprise d'un thème mais aussi l'altération d'une mélodie qui épouse d'autres formes, évoluant au gré du temps et des contextes sociaux, politiques et culturels. Dans les années 1990, alors qu'explosait ce qu'on a appelé la troisième vague du féminisme, les réécritures de Lolita foisonnèrent, adoptant les contours d'une époque qui insistait sur l'indépendance et la prise de pouvoir de la femme dans la société. Deux auteurs, Kim Morrissey dans son recueil Poems for Men who Dream of Lolita (1992) et Pia Pera avec Lo's Diary (1999) offrirent à Dolores Haze la parole dont Humbert l'avait privée dans son manuscrit, en reconstituant son journal de 1947 à 1952. Ces reprises littérales ont ouvert la voie à une veine de réinterprétations, telles que celles d'Emily Präger (Roger Fishbite, 1999) ou de Nancy Jones (Molly, 2000), qui émancipèrent l'adolescente tout en la dotant d'une maturité sexuelle qui ne correspondait pas à celle du roman, mais davantage à la transformation parallèle du personnage en icône populaire, qui excède, voire contredit, le texte auquel elle doit son origine. À l'aube du nouveau millénaire, le mythe de Lolita dépassait les frontières occidentales et devenait un flambeau politique grâce au mémoire de l'écrivain iranienne Azar Nafisi, Reading Lolita in Tehran (2003), traduit en trente-deux langues et en tête des listes des meilleures ventes du New York Times des semaines durant. L'écrivain et critique Erik Morse juge le texte de Nafisi emblématique de cette première décennie du millénaire qui redéfinit la notion de jeunesse féminine 
au Proche et Moyen Orient. Cette jeunesse sera ensuite galvanisée par des figures de proue de l'adolescence telles que Malala Yousafzai, Sihem Habchi, ou le groupe des Femen ${ }^{3}$.

2 Après ces vagues de féminisation puis de politisation, et d'émancipation intellectuelle, que devient Lolita dans les textes de nos écrivains contemporains ? Cet article explore les variations Lolita qu'on a vu fleurir ces dernières années pour tenter de dégager les nouvelles perspectives de la lecture-réécriture de Lolita au $21^{\mathrm{e}}$ siècle. On s'aperçoit qu'à une forme d'instrumentalisation du personnage succèdent des fictions littéraires à part entière, s'éloignant du mythe et des transformations qu'il a subies pour revenir à la substance même du roman de Nabokov, à sa trame, à ses nuances, à sa complexité narrative ou à ses zones d'ombre. Au-delà des amalgames de la presse littéraire qui s'empare bien souvent de l'antonomase «Lolita » dès que se noue dans un roman une relation entre une jeune fille et un homme plus âgé, comme ce fut le cas récemment pour le best-seller de Joël Dicker, La Vérité sur l'affaire Harry Quebert (2012), ces nouvelles lectures de Lolita dialoguent étroitement avec le chef-d'œuvre de Nabokov. Alors âgée de soixante ans, Lolita continue à libérer cette ferveur créative donnant lieu à des hommages inventifs, comme cette fantaisie de Pierre Bayard, qui, en 2014, s'emparant de la théorie des univers parallèles, dans Il existe d'autres mondes, dévoile l'existence de l'original de Lolita, une étudiante américaine de seize printemps et prénommée Annabelle, que Nabokov poursuivit comme un fou sur le campus de Wellesley avant d'écrire le roman de sa vie (Bayard 99-107). Dans Vengeance du Traducteur (2009), Brice Matthieussent réinvente une Dolores Haze qui, dans les marges d'un texte aux intonations kinbotiennes, aurait échappé à ses sinistres amants et réalisé ses rêves hollywoodiens devenant « une ravissante actrice américaine » (Matthieussent 95).

3 La question est alors la suivante : lorsqu'on relit Lolita au $21^{\mathrm{e}}$ siècle, qu'y choisit-on? Est-ce toujours et encore l'iconicité de l'héroïne? Le versant transgressif d'une histoire amour? Ou plutôt une forme de complexité narrative, de richesse thématique et d'extravagance du style? Les trois reprises qui ont retenu notre attention ici sont trois objets littéraires qui se détournent de l'iconicité légendaire et populaire de Lolita pour explorer et réinventer d'autres facettes du roman. Deux d'entre elles offrent le filigrane d'une structure narrative et thématique commune à l'Urtext à travers le destin d'un nouveau Humbert (Nutting et Gaige), tandis que la troisième, reprenant la veine de la filiation littérale, puise dans la mythologie personnelle de l'auteur pour éclairer les zones les plus sombres du roman (Stridsberg).

\section{Tampa, Alissa Nutting (2013)}

4 La double perspective choisie par Alissa Nutting est la transgression et l'inversion des genres. La comparaison repose ici sur la création d'un Humbert Humbert au féminin, qui constitue d'ailleurs un des arguments de la quatrième de couverture ( We had to wait half a century for a female Humbert. It was worth it»). A l'image de l'Urtext nabokovien, la réécriture adopte ici le point de vue du prédateur. Elle se distingue des nombreuses reprises venant combler l'absence du point de vue de la victime dans le roman de Nabokov. Si l'imitation est présente, au sens genettien du terme, c'est l'imitation du scandale et de la transgression. Avant même qu'on ouvre le roman, l'analogie avec Lolita commence par l'histoire de sa distribution, puisque certaines librairies américaines et australiennes ont refusé de vendre cette histoire de pédophile 
en talons, rappelant ainsi la censure dont le chef-d'œuvre de Nabokov fut l'objet soixante ans auparavant ${ }^{4}$.

5 Celeste Price, âgée de vingt-six ans, est un avatar de Humbert, qui dans le seul but de satisfaire son furieux désir sexuel pour de jeunes garçons, devient professeur de littérature dans le collège d'une banlieue américaine. Elisant rapidement une proie en tout point parfaite, elle entretient une relation avec un élève, puis un deuxième, dans un schéma d'obsession dévorante pour son idéal masculin. Nutting décalque donc le schéma nabokovien de liaison illicite entre un professeur de lettres et un jeune esprit à initier, qui a fait, avant Tampa, d'autres émules comme Love in a Dead Language (1999) de Lee Siegel, et plus récemment, L'Attachement, de Florence Noiville (2012).

6 La trame diégétique s'appuie sur les ficelles nabokoviennes. Comme dans Lolita, la narratrice donne une genèse à cette forme de déviance sexuelle. L'épisode Annabel formait dans le texte de Nabokov le maillon téléologique permettant au narrateur de justifier sa différence, et l'épisode «Evan Keller» lui répond dans Tampa. A l'âge de quatorze ans, Céleste a offert sa virginité à ce jeune garçon plus petit qu'elle. La sensation de puissance qu'elle a retirée de leur relation est à l'origine de cette fixation. De même que Humbert déclinait les critères de son désir dans sa célèbre définition de la nymphette ${ }^{5}$, Céleste, en contact chaque jour avec des hordes d'adolescents en ébullition hormonale, jette son dévolu sur un profil précis : l'éphèbe de quatorze ans, au tournant de la métamorphose adulte (" at the very last link of androginy that puberty would permit-undeniably male but not man » [Nutting 25]). Comme pour Humbert, la vie conjugale joue pour la protagoniste le rôle de couverture sociale. Le confort matrimonial se résumait en quelques mots pour Humbert : "a glorified pot-au-feu, an animated merkin " (Nabokov 25); il est la garantie d'un confort luxueux pour une héroïne qui a dû se résoudre à un métier peu lucratif mais qui circule en Corvette. Enfin, tout comme Humbert, Celeste doit consentir à une relation sexuelle avec le père de sa jeune proie pour faciliter sa relation avec le jeune homme. Mais comme dans Lolita, un deus ex machina tragique survient au milieu du roman, qui lui permet de se débarrasser de ce père embarrassant sans se salir les mains.

7 Cette reprise de la trame se situe donc dans le registre parodique. On y lit aussi, dans l'inversion des rôles et dans l'exagération de la reprise, un écho au pastiche d'Umberto Eco, sa nouvelle « Nonita » qui, renversant non pas les sexes, mais les âges du prédateur et de la proie, met en scène un adolescent brûlant d'amour pour une octogénaire décatie :

Nonita, fleur de mon adolescence, angoisse de mes nuits. Pourrais-je jamais te revoir. Nonita. Nonita. Nonita. Trois syllabes, comme une négation faite de douceur. No. Ni. Ta. Puissé-je me souvenir de toi jusqu'à ce que ton image se fasse ténèbres et ta demeure sépulcre.

Je m'appelle Umberto Umberto [...] (Eco 39).

8 Au-delà du jeu avec son propre prénom, le texte d'Eco tirait sa verve humoristique de cette inversion et de la description hyperbolique de cet amour inconditionnel. Le texte de Tampa semble également vouloir rivaliser avec l'humour nabokovien. Il évacue la dimension tragique au profit du rire et de l'exagération, bien que la trame contienne tout son lot de péripéties tragiques, mais Nutting n'explore pas le pathos.

Seulement la farce ne dure jamais qu'un temps, et alors qu'Eco se livrait à un exercice d'une demi-douzaine de pages, dans le cas de Nutting, c'est le format romanesque (293 pages) qui nuance la valeur de cette reprise. L'exagération parodique repose sur une 
simplification des personnages que Nutting réduit à des archétypes monochromes et prévisibles, parfois caricaturaux. Humbert procédait de la même manière-nous pensons à Miss Opposite, Miss Pratt, et en réalité à toute la galerie de ses personnages, Charlotte Haze incluse. Mais que se passe-t-il lorsque cette simplification affecte la narratrice elle-même, comme c'est le cas dans Tampa? Le schéma de l'obsession reste en effet statique tout au long des dix-huit chapitres. Alors que Humbert finit par s'éprendre de la jeune femme adulte, de surcroît enceinte, que devient Lolita, Celeste est résolument attirée par un profil et non un individu. Alors que le collégien enamouré grandit, Celeste le remplace. La divergence majeure avec le roman de Nabokov tient au fait que le lien amoureux du prédateur pour sa victime n'existe pas dans Tampa. La voracité nymphomaniaque dénuée de tout investissement affectif qui caractérise Celeste est une donnée invariable du roman. La déviance sexuelle est par ailleurs si bien assumée par le personnage que les stratégies narratives complexes de Humbert pour couvrir son vice n'auraient aucune pertinence dans ce roman qui explore le versant transgressif en donnant à son héroïne une voix décomplexée par la fureur de ses désirs. Cette radicalisation du désir permet le déploiement d'une verve parodique savoureuse mais fait obstacle à toute forme d'investissement éthique. Enfin, contrairement à Eco qui doublait sa parodie d'un pastiche, la narratrice de Tampa invalide le dicton de Humbert selon lequel on peut toujours compter sur un meurtrier pour une prose alambiquée (« You can always count on a murderer for a fancy prose style »; [Nabokov 2] ]. L'écriture de Céleste n'est pas alambiquée. Elle est aussi vive et goulue que ses pulsions. La première phrase du roman ne trompe pas sur la facture générale $d u$ roman: «I spent the night before my first day of teaching in an excited loop of hushed masturbation on my side of the mattress, never falling asleep » (Nutting 2). La langue de Tampa, contemporaine et hyperbolique, souvent très drôle, a pour ambition de provoquer et choquer le lecteur, non de l'émouvoir. Toute forme de poérotisme nabokovien, pour reprendre le terme de Maurice Couturier, en est exclue. Cette réécriture repose sur l'extrapolation du motif de la transgression. Le potentiel scandaleux du roman n'est-il pas ce qui le définit? Peut-on réécrire Lolita sans en explorer le versant transgressif?

\section{Schroder, Amity Gaige (2013)}

10 C'est la prouesse étonnante réalisée par Amity Gaige dans son roman Schroder, paru la même année que Tampa. "Son cousin littéraire le plus proche est Lolita, sans la pédophilie ", écrit un journaliste du Los Angeles Times dans sa présentation du roman (Brown). Ces mots ont de quoi surprendre. Aurait-on jamais pu réécrire Moby Dick sans la baleine? L'auteur de cette gageure est, elle aussi, américaine et Schroder est son troisième roman. Le roman opère une reprise très serrée du schéma diégétique et $d u$ dispositif narratif sur lesquels s'appuie Lolita. Curieusement, ni l'éditeur, ni la presse ne se sont emparés de cette gémellité littéraire, à l'exception du journaliste du Los Angeles Times et de Kathryn Schulz, qui dans le New York Magazine illustre sa recension par la couverture de Lolita dont auteur et titres auraient été biffés et remplacés par le nom de Gaige et Schroder (Schulz).

11 L'histoire se situe en 2008, dans l'État de New York et est rapportée par un immigré du vieux continent. Eric Schroder et son père ont quitté l'Allemagne de l'Est à la fin des années 70, pour s'installer aux États-Unis alors qu'Eric n'avait pas dix ans. Dans le 
présent de la narration, Eric est un père de famille fraîchement divorcé. Il n'est pas connu sous ce nom, Schroder, mais sous celui de Kennedy, car, à l'âge de quatorze ans, il s'est forgé une fausse identité, optant pour ce patronyme puissamment américain, se glissant petit à petit dans la peau d'un jeune citoyen américain, et entretenant le doute sur sa parenté avec la famille présidentielle. Le choix savamment pensé du pseudonyme et de ses connotations n'est pas sans rappeler celui de Humbert :

And I have toyed with many pseudonyms for myself before I hit on a particularly apt one. There are in my notes "Otto Otto" and "Mesmer Mesmer" and "Lambert Lambert," but for some reason I think my choice expresses the nastiness best (Nabokov 308).

The surname wasn't hard to choose. I wanted a hero's name, and there was only one man I'd ever heard called a hero in Dorchester. A local boy, a persecuted Irishman, a demigod (Gaige 5).

La trame principale reprend de Lolita sa deuxième partie, le voyage en voiture d'un père et de sa fille à travers les États-Unis, dans un contexte de fuite et d'illégalité car Schroder a kidnappé Meadow, sa fille - ici biologique - de six ans, dont il a perdu la garde. La métaphore de la route américaine comme synonyme de liberté est l'une des reprises thématiques, mais c'est aussi et comme dans Lolita, une métaphore tragicomique, car, pour Eric comme pour Humbert, la destination finale du voyage est la prison. Comme dans le roman d'origine, l'aventure prend fin à l'hôpital, où Meadow est admise suite à une crise d'asthme. Confondu grâce à cette hospitalisation, le kidnappeur rédige cette confession depuis sa cellule. Entre temps, dans cette Jaguar dont la banquette arrière est jonchée de canettes de sodas et de papiers de bonbons rappelant le décor nabokovien, on retrouve quelques emprunts à la paranoïa qui dévorait Humbert alors que se succédaient les voitures dans le rétroviseur :

We were mostly through the suburban bottleneck of Albany when I became aware of something in my rearview mirror. A big black shadow of a car that had been lurking several lengths behind. I took a gratuitous left. The car followed. I took a random right. Again the car followed. (Gaige 61)

Le silence et la beauté des paysages de Nouvelle-Angleterre font écho aux pages du voyage de Lolita, à travers la description du paysage, de la pluie et du silence mais aussi à travers l'absence des enfants, qui comme dans Lolita, sont enfermés dans les établissements scolaires tandis que Schroder et Meadow parcourent l'Amérique :

In February, the flora and fauna are dead, the traffic turns the snow the color of tobacco juice, the children are shuttered away in their schools, and the long days are silent. The cats grow wet and skinny, and the rain grows hard and bitter, as if it is not rain but the liquid redistribution of collective conflict; it's a frigid rain, a rain that pricks the skin of any upturned face, a damning rain that makes men eke corks from bottles. O February, you turn our hearts to stone. (Gaige 30-31)

La comparaison ne s'arrête pas à la trame du roman, car le dispositif narratif repose, comme celui de son antécédent littéraire, sur le mode de la plaidoierie d'un narrateur non fiable rédigeant son manuscrit en prison. A l'instar de Lolita, la tonalité juridique des premières pages glisse rapidement vers le genre confessionnel :

What follows is a record of where Meadow and I have been since our disappearance. My lawyer says I should tell the whole story [...]. It's hard not to think about them anyway, my potential listeners. Lawyers. Juries. Fairytale mobs. Historians. But most of all you. You-my whip, my nation, my wife.

Dear Laura. If it were just the two of us again [...]. (Gaige 1) 
15 À cette double énonciation s'ajoute la coloration poétique des lignes de Schroder, à travers le rythme ternaire (« my whip, my nation, my wife ») et la métaphore utilisée à l'encontre du jury («Fairy-tale mobs»), qui rappellent la poésie allitérative et rythmique de Humbert et ses allégations contre le jury via l'intertexte de Poe (« exhibit number one is what the seraphs, the misinformed, simple, noble-winged seraphs, envied. Look at this tangle of thorns " [Nabokov 9]). Ajoutons que le prénom de son épouse, Laura, resserre le lien à Nabokov, dont le roman posthume, The Original of Laura, paraît en 2009, soit quelques années seulement avant Schroder.

Comme dans Lolita, le récit procède par grandes analepses: Schroder revient épisodiquement sur un passé douloureux qui crée la chaîne causale justifiant le mensonge gouvernant sa vie, notamment la perte de sa mère, qui disparaît, comme celle de Humbert, dans ses premières années :

Mine was a tale that, by certain lights, was the truest thing I had ever written. It involved the burdens of history, an early loss of a mother, a baseless sense of personal responsibility, and dauntless hope for the future. (Gaige 5)

Le portrait du narrateur s'étoffe à mesure que le manuscrit s'écrit et fait revivre dans l'esprit du lecteur les images de l'exil mélancolique que dépeint Humbert dans les premiers chapitres de Lolita, à savoir une vie de dilettante et de dandy émigré. Schroder est l'avatar allemand du jeune Humbert parisien qui publiait de tortueux essais universitaires et débatait de cinématographie avec les expatriés russes :

In my free time, I worked erratically on my research (see page 15) and played soccer with a bunch of foreign transplants on a hill we borrowed from the College of Saint Rose. (Gaige 9)

Paris suited me. I discussed Soviet movies with expatriates. I sat with uranists in Deux Magots. I published tortuous essays in obscure journals. (Nabokov 16)

Enfin, le thème du double qui structure la narration de Lolita se retrouve dans Schroder à travers l'oscillation pronominale entre la première et la troisième personne. Grâce à une reprise de ce schéma, Gaige traduit la complexité d'un moi tentant de se disculper des accusations qui pèsent sur lui, comme le faisait Humbert :

For the record: the groom never told the bride that he was related to the Kennedys of presidential fame. This has been reported in the papers, and the groom categorically denies it. [...] The groom will admit that once or twice late at night with his female peers at Mune College, he did not sufficiently debunk the rumor of himself as a second cousin twice removed to the Hyannis Port Kennedys. (Gaige 13) But let us be prim and civilized. Humbert Humbert tried hard to be good. Really and truly, he did. He had the utmost respect for ordinary children, with their purity and vulnerability, and under no circumstances would he have interfered with the innocence of a child, if there was the least risk of a row. (Nabokov 19)

La duplicité se fait jour également dans la scission intérieure du personnage, liée aux langues qu'il parle. L'idiome américain a été parfaitement intégré par cet émigré, mais son manuscrit est semé de courtes phrases en allemand, la langue de l'enfance qui, ici, semblent exprimer le refoulé, tandis que des interjections françaises et des locutions latines rappellent le pédantisme de son précurseur.

Cette préciosité narrative permet à Gaige de revisiter et de développer de petites manies proto-postmodernes de Nabokov. Son héros se complaît dans les digressions et notes de bas de page kinbotiennes sur la typologie du silence, objet de ses recherches ${ }^{6}$. Elle fait également écho à l'hybridité du texte de Nabokov en incluant dans le texte un scénario que Schroder écrit pour sa femme, qui rappelle le poème à la gloire de Lolita 
que Humbert insère dans son manuscrit. Le roman déploie par ailleurs différents modes d'écritures et réécritures du réel, du fait divers aux infos télévisées, du journal à la littérature, qui l'associent à l'hybridité générique du roman de Nabokov.

Si le héros de ce livre n'est pas pédophile, la narration est soumise au même vacillement psychologique que celui qui caractérisait le manuscrit de Humbert. La ressemblance n'est plus à démontrer lorsque, cédant à une forme de pathologie narrative incontrôlée, Schroder répète la phrase «I let you down » sur quatre pages (255-258), développant littéralement l'instruction de Humbert à son typographe dans le plus court chapitre de Lolita :

This daily headache in the opaque air of this tombal jail is disturbing, but I must persevere. [...]. Don't think I can go on. Heart, head-everything.Lolita, Lolita, Lolita, Lolita, Lolita, Lolita, Lolita, Lolita, Lolita, Lolita. Repeat till the page is full, printer. (Nabokov 109)

Le titre retenu par Gaige, qui, à l'inverse du roman de Nabokov, est celui du protagoniste masculin, donne la clé de la modulation opérée ici. Contrairement à nombre de reprises de Lolita se focalisant sur le personnage éponyme du roman, la lecture se concentre exclusivement sur la profondeur du personnage de Humbert. Le potentiel affectif du personnage de Meadow, à l'instar de celui de Lolita, n'est pas exploré 7 . Du roman de Nabokov, cette réécriture reprend davantage la complexité de la narration et de la caractérisation que le versant polémique. Une référence à Nabokov, qui se fait sur le mode allusif par l'intermédiaire de la petite fille, nous éclaire sur la position de Gaige :

"When you grow up. What do you want to be famous for? Everybody wants to be famous for something."

"I want to be a lepidopterist." Not unkindly, Meadow added, "Lepidopterists study butterflies."

"You're not going to be famous for that." (Gaige 137)

Ce that souligné par les italiques traduit l'ironie de l'auteur sur la raison de l'immense célébrité de Nabokov, qui est précisément le thème qu'elle ne reprend pas ici. Car la relation de Schroder à sa fille est celle de l'amour fou, mais de l'amour paternel. L'absence de relation transgressive permet à Gaige de rendre justice à un roman que certaines institutions continuent à écarter des programmes en raison de son contenu sulfureux.

\section{Darling River, Les Variations Dolorès, Sara Stridsberg. (2010)}

La relation sexuelle illicite est également absente du texte de Sara Stridsberg, bien qu'une sexualité consentie et protéiforme y soit omniprésente. Cette troisième relecture est la plus littérale. Comme en témoigne l'appendice titulaire de ce roman (Les variations Dolorès) la présence de la Lolita nabokovienne y est pleinement assumée. Darling River est un texte entièrement structuré par la figure de Dolores, que Stridsberg refaçonne pour la faire coïncider avec l'univers personnel qui habite sa fiction.

Le roman repose sur une architecture complexe de cinq trames distinctes revenant tour à tour, sans ordre apparent, dans les cinq blocs narratifs intitulés "Le Destin ", «Le Temps », «Le Miroir », «La Maladie », et pour finir, «La Solitude » qui n'occupe que trois pages. Trois de ces trames, intitulées «Darling River (Lo)», «Le Livre des 
Morts ", et «Le Jardin des Plantes », reprennent des thématiques purement nabokoviennes tandis que les deux suivantes "Sur la Mappemonde maternelle » et «Encyclopédie » viennent créer des liens entre les mythes littéraires et les sujets qui hantent l'écriture de Sara Stridsberg.

L'extraction diégétique la plus proche du texte-source est celle du « Livre des Morts ». Contrairement aux deux autres romans qui reposaient sur l'imitation, cette section comble une béance du récit de Nabokov, celle des dernières journées de Lolita et de sa disparition, le jour de Noël 1952 à Gray Star. Ce cas, envisagé par Genette dans son traité de la réécriture, constitue précisément « une continuation elliptique : chargée de combler une lacune ou une ellipse médiane sur un mode analeptique, remontant de cause en cause, jusqu'à un point de départ » (Genette 1982, 242). En effet, Stridsberg, partant du 25 décembre 1952, jour de l'accouchement et de la mort de Lolita et de sa fille, suit une linéarité à rebours de la chronologie. La première image est celle d'une Dolores, encore pleine d'espoir, s'apprêtant, sur le lit de la maternité qui est aussi son futur lit de de mort, à donner naissance à son enfant :

La sage-femme passe le rasoir sans penser qu'entre les jambes de Dolores toujours hâlées et gluantes et tremblantes et nues enfle une bulle de chewing-gum rose et que dans sa tête un parfum de fraises et de soleil et d'espérances explose. (Stridsberg 34)

L'hypotexte nabokovien est assimilé et comme réverbéré par Stridsberg, car la fusion entre le motif symbolique de l'innocence, la «bulle de chewing-gum rose », et celui du sang et de la mort, nous renvoie au meurtre de Quilty dans les derniers chapitres de Lolita :

I hit him at very close range through the blankets, and then he lay back, and a big pink bubble with juvenile connotations formed on his lips, grew to the size of a toy balloon, and vanished. (Nabokov 304)

Les passages suivant cette première scène retracent le voyage à Gray Star, remontant le temps. Dolores, sur la banquette arrière d'une voiture conduite par Richard Schiller, fait le deuil de ses rêves hollywoodiens pour songer désormais à l'innocence et la blanche pureté de l'Alaska. Mais, dans une tonalité propre à Stridsberg, son corps est de plus en plus violenté par la maternité tandis qu'une écriture de la morbidité se concentre sur les sensations de fièvre, de froid et de douleurs corporelles. Au travers des récits de cauchemars qui se succèdent, le texte produit quelques allusions à la vie de Dolores avant Richard, à son beau-père (Stridsberg 34, 112, 207 passim) et ses cadeaux, comme ce livre consacré au chant des oiseaux qui ne lui a jamais plu (Stridsberg 304) et à sa mère disparue dans un accident de voiture (Stridsberg 206).

Le récit est écrit à la troisième personne mais il adopte le point de vue de Dolores et offre ainsi au lecteur un accès à son intimité psychique, grâce à la technique de la transvocalisation qui consiste à transférer le point de vue sur un autre personnage que celui de l'hypotexte (Genette 1982,412). Mais Stridsberg offre une reprise moins littérale que personnelle et donne à Dolores un espace d'expression qui lui permet d'échapper à la lecture stéréotypée du personnage de l'adolescente américaine. Le prénom retenu par Stridsberg, "Dolores", pas une seule fois Lolita, renforce l'autonomie d'un personnage qui n'est ici pas soumis à la médiation et la transformation opérées par le regard et l'écriture du personnage masculin. Dans La Vengeance du traducteur, de Brice Matthieussent, une note de bas de page donnant la voix à Dolores Haze revient sur l'importance du prénom de l'état civil aux dépens des surnoms que déclinait Humbert, Lolita restant le pire de tous : 
Tu peux bien m'appeler ta Dodo, ta Dora, ta Dollie, ta Loli, ta Lola, ta Loli, mais jamais Lolita, c'est beaucoup trop vulgaire. Je suis Dolores pour l'état civil et pour toi tout ce que tu voudras, sauf Lolita [...].

Plusieurs fois pourtant je t'avais dit que je détestais ce prénom, qu'il me ramenait vers une époque malheureuse de mon existence. (Matthieussent 143)

Comblant les béances, le texte permet au lecteur d'accéder à ce que Humbert regrettait de n'avoir qu'entr'aperçu de Lolita - «a twilight, and a palace gate - dim and adorable regions " (Nabokov 284) ${ }^{8}$, déroulant les réminiscences heureuses, des rêves d'avenir, mais aussi, une série de cauchemars, qui incluent souvent celui qui est appelé dans le texte son «beau-père ", ainsi qu'un leitmotiv sur le mystère qui a entouré la mort de sa mère :

Maman est sortie dans la rue en courant avant d'être écrasée par une voiture. Quand je suis rentrée, son corps avait déjà été enlevé. Je ne sais même pas où elle est enterrée (Stridsberg 206).

«Où est enterrée maman?»

«Qui l'a écrasée?»

«Maman...»

«Mama...»

«Mami... ». (Stridsberg 214)

31 La deuxième sous-partie directement liée au texte de Nabokov est intitulée «Darling River (Lo) ». C'est la plus volumineuse dans l'économie du roman, auquel elle donne son titre et son centre de gravité narratif. Cette trame présente une deuxième Dolores, qui doit son prénom au roman d'un Nabokov identifié allusivement, comme chez Gaige, par sa passion pour la lépidoptérologie, ainsi que par ses origines russes :

Pendant mon enfance, mon père était passionné par un écrivain russe en exil, par ailleurs collectionneur de papillons, et s'était du même coup servi d'un de ses romans pour me baptiser [...]. Dolores signifie douleurs et je songe que cela pourrait tout aussi bien vouloir dire roses. (Stridsberg 23)

C'est une filiation de texte en texte qui s'opère ici et qui, créant un nouveau personnage distinct de la Lolita de Nabokov, ne cesse pourtant de renvoyer à son antécédent, le texte de Nabokov. Il est fait allusion à l'héritage littéraire de Lolita dans une scène du roman où cette nouvelle Dolores cherche, à la Bibliothèque Nationale, "un livre qui parle de moi » (Stridsberg 85), et qui lui permettrait de déchiffrer les mystères de sa destinée: "Je cherche des messages secrets dissimulés dans les volumes anciens» (Stridsberg 85).

33 Comme pour Schroder, cette trame reprend le schéma d'un père et de sa fille sur la route en l'absence de la mère et d'une relation incestueuse. D'autres reprises thématiques jalonnent leurs voyages, comme le tennis et la jupette de Lo ou les bandes dessinées. Le père de Lo est un chasseur, enchanté par l'alcool et les prostituées. Le voyage, comme celui de Humbert et Lo, n'a pas de réelle destination :

Les saisons défilaient et nous roulions. Nous roulions dans la canicule, dans la neige dans les cendres, à travers les bois de bouleaux [...]. (Stridsberg 13).

Nous nous promenions en cercles toujours plus concentriques. (Stridsberg 17)

Comme dans Lolita, et comme dans la reprise de Gaige, le voyage n'est pas synonyme de liberté car il dessine les contours d'un enfermement psychique et d'une isolation sociale: «Nous sommes toujours seuls» écrit la narratrice (Stridsberg 15). Ces circonvolutions sont ternies par une pellicule de décrépitude qui recouvre le paysage et qui va s'en prendre bientôt au corps de Dolores. Car ce corps, toujours vêtu de socquettes blanches, de robes d'enfance de plus en plus serrées, se couvre de vésicules, 
de taches noires et est bientôt avili par une maladie mystérieuse, que tente de soigner un médecin de Saint-Pétersbourg. Ajoutons que cette Dolores n'est pas une jeune fille innocente. A moitié alcoolique, le soir, elle rencontre ses nombreux amants au bord de la rivière pendant que son père courtise des prostituées.

Derrière l'hommage à Nabokov, c'est donc un contre-récit qui se dessine. Il ne reste rien, ou presque, de la figure iconique de Lolita. Ce sont les violations auquel Humbert a soumis une enfant qui sont, ici, le fruit d'une extrapolation créative. Ce chant de l'expérience exhibe la dégradation du corps féminin. À la fin du livre, Dolores atteint bientôt l'âge de 36 ans, sans qu'on ait vu le temps passer, et connaît les pires affres d'une féminité morbide: avortement, maladies, enlaidissement et décrépitude. La symbolique et les motifs nabokoviens, toujours très présents, sont soumis à cette contamination mortifère, comme ce papillon mort que Lo imagine sortir de sa bouche : «Je me regarde si longtemps dans la glace que j'ai l'impression qu'un papillon va sortir de ma bouche. Un papillon à tête de mort » (Stridsberg 86).

Enfin, une troisième trame revient sur l'anecdote génétique du singe du Jardin des Plantes, que Nabokov donnait comme source d'inspiration du roman (Nabokov 311). Dans cette sous-partie, un scientifique du jardin parisien, socialement isolé et repoussé par les femmes, cherche par tous les moyens à faire dessiner une femelle chimpanzé, la captive et la victime de son obsession. Chaque chapitre est le récit d'un mois d'expériences infructueuses, au terme desquelles le singe finit par se soumettre et produire une esquisse représentant le grillage de sa cage. Mais à travers cette extrapolation fictive de l'anecdote génétique, Stridsberg offre une nouvelle interprétation du motif de la captivité qui structure Lolita et développe la métaphore du désir masculin de recréer un idéal féminin.

Or, ce motif traverse toutes les trames narratives : le corps de la femme dans ce livre est sans cesse soumis au regard de l'homme. Si Stridsberg donne voix à deux Dolores, ce n'est pas tant pour émanciper l'adolescente que pour montrer les conséquences de cette réification de la figure féminine du roman. La première Dolores est brisée par le deuil progressif de ses rêves hollywoodiens avant de mourir dans l'enfantement, tandis que la seconde est hantée par la peur que sa maladie n'écarte ses amants. S'il est un hommage à Nabokov, le roman de Stridsberg est également un contre-récit qui s'inscrit dans la réflexion sur la féminité esquissée par les réécritures de Lolita depuis la fin du $20^{e}$ siècle. Mais contrairement aux réécritures qui le précèdent, il multiplie les réflexions à travers l'entrelacement des trames narratives et des personnages. La réflexion alors générée ne s'arrête pas à l'image de la femme-enfant, mais l'étend à toutes les figures féminines du roman, Dolores, Lolita, les prostituées que rencontre le père, mais aussi les figures maternelles et solitaires, qui constituent la quatrième trame de cette reprise.

38 À l'occasion de ce soixantième anniversaire, nous observons que même si elle a atteint un âge canonique, la fille-enfant de Nabokov n'a pas pris une ridule, car elle continue de prospérer à travers les textes qu'elle n'en finit pas d'engendrer. On ne pourra s'empêcher de noter l'origine féminine d'un grand nombre de ces relecturesréécritures, ce qui, dans les années quatre-vingt dix, a pu s'expliquer par la volonté de rendre justice à un personnage féminin séquestré par la prose masculine. Mais ces nouvelles lectures dépassent la revendication féministe. Si Nutting, dans l'inversion des genres qu'elle opère, offre une imitation ludique du potentiel littéraire scandaleux de la pédophilie, Gaige et Stridsberg s'emparent d'une facette sombre de chacun des 
personnages principaux pour en éclairer la complexité. Ce que ces trois lecturesréécritures soulignent, plus d'un demi-siècle ayant passé, c'est la richesse prismatique de l'ouvrage-source. Dans un entretien, Sara Stridsberg compare Lolita à une galerie des glaces (2011). La profondeur du roman de Nabokov l'apparente, dit-elle, à jeu de miroirs, où Lolita et Humbert, diffractés, génèrent une série infinie de réflections, d'images, parfois d'ombres, mais aussi de surfaces sur lesquels les écrivains et héritiers littéraires continuent de projeter leurs lectures du $21^{\mathrm{e}}$ siècle.

\section{BIBLIOGRAPHIE}

Artus, Hubert. « Stridsberg se paye le mythe de Lolita dans Darling River ». Rue 89. 11 mai 2001, consulté le 31 mai 2016.

<http://rue89.nouvelobs.com/blog/cabinet-de-lecture/2011/05/11/sara-stridsberg-se-paye-lemythe-de-lolita-dans-darling-river-203210>

Beigbeder, Frédéric. Au secours, pardon. Paris : Livre de Poche, 2008.

Brown, Janelle. « A Real Impostor's Tale Inspires Fascinating Fiction in Schroder ». Los Angeles Times, 21 février 2013, consulté le 31 mai 2016.

< http://articles.latimes.com/2013/feb/21/entertainment/la-ca-jc-amity-gaige-20130224>

Couturier, Maurice. Le Rapt de Lolita. A paraître.

Dicker, Joël. La Vérité sur l'affaire Harry Quebert. Paris : Fallois-L'Âge d'homme, 2012.

Eco, Umberto. « Nonita ». In Pastiches et Postiches. Trad. Bernard Guyader. Paris : Messidor, 1988, 39-45.

Gaige, Amity. Schroder. New York : Twelve, 2013.

Genette, Gérard. Palimpsestes. Paris : Editions du Seuil, 1982.

---. « L’Autre du même », dans Figures IV. Paris : Seuil, 1999, 101-107.

Jones, Nancy. Molly. New York : Crown Publishers, 2000.

Loison-Charles, Julie. « Nabokov et la censure ». Miranda [Online] 15 (2017). 06 octobre 2017. https://miranda.revues.org/11223

Matthieussent, Brice. La Vengeance du traducteur. Paris : P.O.L., 2009.

Morrissey, Kim. Poems for Men who Dream of Lolita. Regina : Coteau Books, 1989.

Morse, Erik. « A Portrait of the Young Girl : On the 60th Anniversary of Nabokov's Lolita. An Interview Series ». Los Angeless Review of Books, January 13 2015. Consulté le 31 mai 2016.

< https://lareviewofbooks.org/article/portrait-young-girl-60th-anniversary-nabokovs-lolitapart-iv-interview-series/> .

Nabokov, Vladimir. The Annotated Lolita. Dir : Alfred Appel. New York : Vintage, 1991.

Nafisi, Azar. Reading Lolita in Tehra n: A Memoir in Books. New York : Random House, 2003.

Noiville, Florence. L'attachement. Paris : Editions Stock, 2012. 
Nutting, Alissa. Tampa. New York : Harper and Collins Publisher, 2013.

Pera, Pia. Lo's Diary. Trad. Ann Goldstein. New York : Foxrock, 1999.

Präger, Emily. Roger Fishbite. London : Chatto \& Windus, 1999.

Schulz, Kathryn. « Kathryn Schulz on Amity Gaige's Novel Schroder ». New York Magazine, 18

février 2013. Consulté le 31 mai 2016.

http://www.vulture.com/2013/02/kathryn-schulz-on-schroder-by-amity-gaige.html

Siegel, Lee. Love in a Dead Language. Chicago : University of Chicago Press, 2000.

Stridsberg, Sara. Darling River- Les Variations Dolorès. Trad. Jean-Baptiste Coursaud. Paris : Stock, 2011.

Turine, Natalia (dir.). Lolita, Variations sur un thème, recueil de nouvelles de Claire Berest, Philippe Besson, Catherine Locandro, Nicolas Rey, Christophe Tison, Emilie Frèche, Murielle Magellan, Emmanuelle Richard, Richard Millet, Maurice Couturier. Paris : Louison éditions, 2016.

Williams, John. « Desperate Dad: Amity Gaige Talks About 'Schroder'« . The New York Times, 6 février 2013. Consulté le 31 mai 2016.

<http://artsbeat.blogs.nytimes.com/2013/02/06/desperate-dad-amity-gaige-talks-aboutschroder/>

\section{NOTES}

1. Les écrivains Claire Berest, Philippe Besson, Catherine Locandro, Nicolas Rey, Christophe Tison, Emilie Frèche, Murielle Magellan, Emmanuelle Richard, Richard Millet, Maurice Couturier, acceptèrent de revisiter l'œuvre de Nabokov dans une nouvelle, et, sollicités par la maison d'éditions Louison, ont signé une nouvelle dans cet ouvrage préfacé par Maurice Couturier (Turine [dir.], Lolita, Variations sur un thème, Paris : Louison éditions, 2016).

2. Dans son court article "L'autre du même ", Gérard Genette reprend la métaphore musicale de la variation pour évoquer les problématiques liées à la réécriture en littérature et en musique, et en particulier celle de la double notion de différence et d'identité engendrée par toute reprise (Genette 1999, 101-107).

3. L'analyse de cette nouvelle pensée de l'adolescence féminine est amorcée par Erik Morse dans le numéro spécial consacré à l'anniversaire de Lolita dans le Los Angeles Review of Books. La quatrième section de ces entretiens dresse notamment un inventaire des différents contextes qui ont jalonné la réécriture de Lolita dans la deuxième moitié du $20^{\text {e }}$ siècle: < https:// lareviewofbooks.org/article/portrait-young-girl-60th-anniversary-nabokovs-lolita-part-ivinterview-series/ $\geq$

4. On se reportera ici au travail de Julie Loison-Charles sur Lolita et la censure dans ce même numéro, et notamment la question de son interdiction dans certaines bibliothèques américaines.

5. « Now I wish to introduce the following idea. Between the age limits of nine and fourteen there occur maidens who, to certain bewitched travelers, twice or many times older than they, reveal their true nature which is not human, but nymphic (that is, demoniac); and these chosen creatures I propose to designate as "nymphets" ». (Nabokov 16)

6. Dans les entretiens, Gaige se défend curieusement de la comparaison avec Lolita mais cite Pale Fire comme source d'inspiration. Voir l'entretien réalisé par John Williams et paru dans le New York Times : « Desperate Dad: Amity Gaige Talks About "Schroder” » 6 février 2013.

7. Cette réécriture prend donc le contrepied des nombreuses reprises publiées dans les années 1990, comme celles de Pia (Lo's Diary, 1999), Nancy Jones (Molly, 2000), d'Emily Präger (Roger Fishbite, 1999) puis plus récemment de Frédéric Beigbeder, (Au Secours Pardon, 2008), qui 
s'efforcent de donner la voix et la profondeur humaine qui manquaient au personnage de l'adolescente dans le manuscrit de Humbert Humbert.

8. «I simply did not know a thing about my darling's mind and that quite possibly, behind the awful juvenile clichés, there was in her a garden and a twilight, and a palace gate-dim and adorable regions which happened to be lucidly and absolutely forbidden to me, in my polluted rags and miserable convulsions ». (Nabokov 284)

\section{RÉSUMÉS}

Lorsqu'on réécrit Lolita au $21^{\mathrm{e}}$ siècle, qu'en retient-on? Est-ce l'iconicité de son héroïne? Le versant transgressif d'une histoire amour? Ou sa complexité narrative et thématique ? Après être revenu sur les nuances féministes et politiques qui ont teinté nombre de réécritures de Lolita au tournant du siècle, le présent article se penche sur les plus récentes reprises pour tenter de dégager les nouvelles perspectives de la lecture-réécriture de Lolita aujourd'hui. L'analyse porte en particulier sur trois romans entretenant une relation étroite avec le texte original. Tandis que Tampa, d'Alissa Nutting, exploite le thème de la transgression en recréant un Humbert Humbert au féminin, Amity Gaige, dans Schroder (2013), exclut le thème de la pédophilie, pour revisiter le thème de l'exil et du road trip américain d'un père et de sa fille. Enfin, l'écrivain suédoise Sara Stridsberg, dans Darling River, Les Variations Dolores, offre une relecture fragmentée, multiple et personnelle de Lolita qui, en poursuivant des fils esquissés par Nabokov, éclaire encore davantage la complexité de son original.

When creating a new version of Lolita in the $21^{\text {st }}$ century, what do authors retain from Nabokov's masterpiece? Is it the myth and the icon or is it the diegetic thread? Is it the extravagant prose or the transgression and taboo? After reviewing the feminist or political nuances that tinted many a rewriting of Lolita at the turn of the century, the following article seeks to examine the more recent perspectives offered by the rewriting of Nabokov's masterpiece in the $21^{\text {st }}$ century. The analysis focuses in particular on three novels that claim close affinities with Lolita. Alissa Nutting's Tampa (2013) exploits the theme of transgression by creating a feminine Humbert craving for sex with teen boys, while Gaige excludes pedophilia to focus instead on the theme of European exile and of the illicit father-and-daughter roadtrip. Darling River, Les Variations Dolores (2011) by Swedish author Sara Stridsberg is an intensely personal and multilayered rewriting of Lolita that, along with Gaige and Nutting, powerfully illustrates how Lolita's avatars still shed light on the complexity of their original.

\section{INDEX}

Mots-clés : Lolita, mythe, réécriture, variation, hommage, parodie, pastiche, palimpseste, transgression, tabou, pédophilie, road novel

Keywords : Lolita, myth, rewriting, variation, tribute, parody, pastiche, palimpsest, transgression, taboo, pedophilia, road novel 


\section{AUTEURS}

\section{YANNICKE CHUPIN}

Maître de conférences.

Université de Cergy-Pontoise /Agora (EA 7392)

Yannicke.chupin@u-cergy.fr 